Therefore, even after one complete sound wave there will be already a clearly marked selection of the " in-tune" group of resonators.

Taking now the physiological effects into consideration, the threshold factor will definitely rule out all amplitudes below a certain value, so that after a certain number of sound waves have entered the ear the amplitude of the "in-tune" resonators will be the first to rise above the threshold and will cause impulses to pass up the auditory nerve. A little later a larger number of resonators will have reached amplitudes above the threshold, so that there should be a gradual increase in the number of resonators in active response, until the full steady value is reached. Calculation shows that the "in-tune" resonator should attain 90 per cent. of its final amplitude in eight vibrations. On the resonance theory one would therefore expect a gradual rise in the sound intensity occurring in a time interval of the order of Io-20 vibrations of the incoming sound waves (i.e. $\mathrm{I} / 25^{-\mathrm{I} / \mathrm{I} 2} \mathrm{sec}$. for middle $\mathrm{C}$ ), and not a fall as $\mathrm{Mr}$. Ackermann has suggested in his letter. It would seem that this effect is responsible for the absence of roughness at the commencement of a tone due to the imperception of the transient vibrations of "out-of-tune" resonators.

Now if the rise of sound intensity is a gradual one, what, it may be asked, is the mode of perception of a tone which starts with large amplitude and gradually diminishes as it goes on-e.g. a piano note? In such a case it would seem that after a very few vibrations, the swings of the resonators must reach such an amplitude that their motion is perceived. In this case, then, the vibration of "out-of-tune" resonators makes itself perceived because the auditory nerve fibres are taking up responses before there have been sufficient incoming sound waves to damp out the "out-of-tune" resonators. It would seem to be this effect which gives the transient harshness to the commencement of a piano note, causing it to sound to the ear as if it started with a consonant.

King's College, Cambridge.

H. HARTRIDGE.

\section{An Experimental Towing-tank used by Benjamin Franklin.}

IN the "Calendar of Industrial Pioneers" in NATURE, May 6, p. 598, relative to the anniversary of William Froude, your correspondent says: "His (Froude) work led to the construction by the Admiralty of the experimental tank at Torquay, the first of its kind ever built."

It will be doubtless interesting to readers of NATURE to have it brought to their attention that Benjamin Franklin in his many and varied investigations in philosophical subjects investigated, to some extent, the difference of navigation in shoal and deep water. In a letter written to Dr. John Pringle, May Io, I768, he gives the results of experiments made along these lines. The letter tells of how, during a trip with Dr. Pringle in Holland, it was brought to their attention that the treckschuyt in one of its trips went slower than usual, due, as the boatmen explained, to the water in the canal being low. After his return to England, not being entirely satisfied with the boatman's explanation, Franklin questioned the Thames river watermen and found them all agreeing as to fact, but differing widely in expressing the quantity of the difference. He, therefore, designed the following experiment, which in its nature is a forerunner of the modern towingtank. I quote from his letter:
" I provided a trough of planed boards fourteen feet long, six inches wide, and six inches deep in the clear, filled with water within half an inch of the edge, to represent a canal. I had a loose board, of nearly the same length and breadth, that, being put into the water, might be sunk to any depth, and fixed by little wedges where I would choose to have it stay, in order to make different depths of water, leaving the surface at the same height with regard to the sides of the trough. I had a little boat in form of a lighter or boat of burden, six inches long, two inches and a quarter wide, and one inch and a quarter deep. When swimming, it drew one inch water. To give motion to the boat, I fixed one end of a long silk thread to its bow, just even with the water's edge; the other end passed over a well-made brass pulley of about an inch diameter, turning freely on a small axis; and a shilling was the weight. Then, placing the boat at one end of the trough, the weight would draw it through the water to the other.

"Not having a watch that shows seconds, in order to measure the time taken up by the boat in passing from end to end, I counted as fast as I could count to ten repeatedly, keeping an account of the number of tens on my fingers. And as much as possible to correct any little inequalities in my counting, I repeated the experiment a number of times at each depth of water, that I might take the medium. And the following are the results :

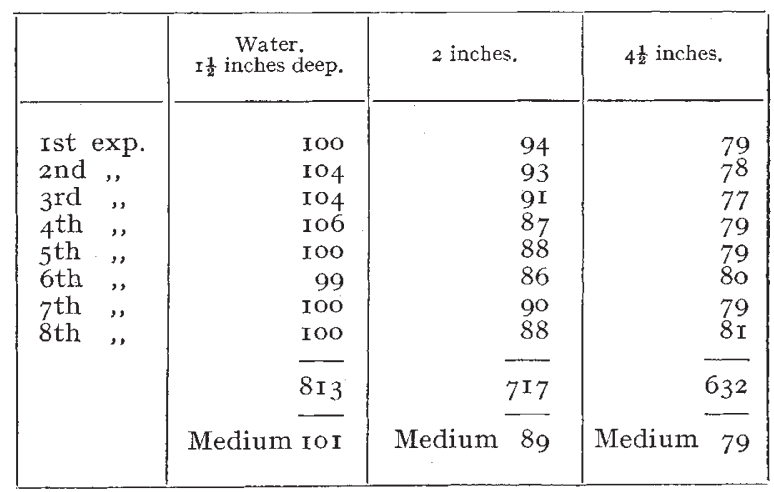

U.S. Coast and Geodetic Survey

Paul C. Whitney. Washington, D.C., May 22.

\section{An Experimental Confirmation of the Kinetic and Molecular Theories of Magnetism.}

CURIE's law states that ferromagnetics above the critical temperature behave in such a way that the susceptibility $(k)$ is inversely proportional to the absolute temperature $(T)$, in short, that the product $k . T$ is a constant. The physical meaning of this law is that when the molecular magnets have complete freedom of rotational movements, the energy of magnetisation is then only opposed by the energy of thermal agitation and, consequently, any given state is a state of equilibrium.

Below the critical temperature complications introduced by the mutual magnetic actions of the molecules, one on the other, and by the approach to a saturation limit have obscured any such simple law. It is, however, possible to eliminate, or allow for, the effects of these disturbing factors and to make experiments, under hysteresis-free conditions, upon the variation of susceptibility with temperature. Experiments of this kind have been carried out on

$$
\text { NO. } 2748 \text {, VOL. I IO] }
$$

